\title{
Is a convex plane body determined by an isoptic?
}

\author{
ÁrpÁd KurusA*
}

\begin{abstract}
We prove that two convex bodies coincide if they have a common isoptic with the same angle $\nu \in(0, \pi)$ so that $1-\nu / \pi$ is an irrational or rational number with even numerator in its lowest terms. In the remaining cases we prove that two convex polygons with a common isoptic coincide, and a common isoptic of different rotationally symmetric bodies is a circle.
\end{abstract}

\section{Introduction}

The set of points where a compact convex domain $\mathcal{B}$ with nonempty interior (such domains are called convex bodies, the strictly convex ones are called ovals or ovoid bodies, and the ones bounded by polygons are called convex polygonal bodies or simply convex polygons in this paper) subtends a constant angle $\nu \in(0, \pi)$ is called the $\nu$-isoptic of the convex body $\mathcal{B}$. The $(\pi / 2)$-isoptic is called orthoptic.

A short list of isoptics of some important, but not necessarily convex plane curves can be found in [10]; further results on isoptics and the strongly related inner isoptics can be found in [7], [5].

This article considers the following question:

Is a convex plane body determined by a $\nu$-isoptic of it?

In 1950 Green [1] proved that if the $\nu$-isoptic of a convex body $\mathcal{D}$ is a circle, then $\mathcal{D}$ must be a disc provided $1-\nu / \pi$ is an irrational or rational number with even numerator in its lowest terms. If the numerator is odd, then there are continuum many bodies not even similar to each other with that circle as their $\nu$-isoptic.

In $1971 \mathrm{~W}$. Wunderlich [9] showed the existence of continuum many bodies in all ellipses $\mathcal{E}$, which have numerical excentricity near 1 , so that the $\nu$-isoptic of all

AMS Subject Classification (2000): 0052, 0054.

Key words and phrases: visual angle, isoptics, shape recognition, shadow picture, distinguishability of plane convex bodies.

* Supported by the TAMOP-4.2.1/B-09/1/KONV-2010-0005 project.

Beiträge zur Alg. und Geom., 53 (2012), 281-294.

(c) Á. Kurusa 
these bodies are $\mathcal{E}$ provided $1-\nu / \pi$ is rational with odd numerator in its lowest terms.

We provide uniqueness results to question (1.1) for ellipses, for special angles, for convex polygons and for rotationally symmetric convex bodies.

\section{Preliminaries}

Let the boundary and the $\nu$-isoptic of a convex body $\mathcal{B}$ be denoted by $\partial \mathcal{B}$ and by $\mathcal{B}^{\nu}$, respectively. A convex body $\mathcal{G}$ is said to be a $\nu$-ghost of the convex body $\mathcal{B}$ if $\mathcal{G}^{\nu} \equiv \mathcal{B}^{\nu}$.

Let $\min _{\mathcal{B}} \alpha$ denote the minimum of the angles at the singular points of $\partial \mathcal{B}$. If $\partial \mathcal{B}$ does not have singular points, i.e., $\mathcal{B}$ has differentiable border, then we define $\min _{\mathcal{B}} \alpha:=\pi$. If $\nu \geq \min _{\mathcal{B}} \alpha$, then the $\nu$-isoptic of $\mathcal{B}$ is a closed curve, but it is not connected if $\nu<\min _{\mathcal{B}} \alpha$.

If $\min _{\mathcal{B}} \alpha=\pi$, then $\mathcal{B}^{\pi}=\partial \mathcal{B}$, hence the $\nu$-isoptic is convex if $\nu$ is sufficiently close to $\pi$. In the other hand the $\nu$-isoptics of a "sufficiently depressed" ovals are concave for sufficiently small angles $\nu[6]$.

Nevertheless, all isoptics are star-shaped with respect to any point in the convex body $\mathcal{B}$, because on every straight line that passes through $\mathcal{B}$ the visual angle of $\mathcal{B}$ is strictly monotone decreasing as the point is moving away from the chord in which the straight line intersects $\mathcal{B}$. This observation also proves that $\mathcal{B}^{\nu}$ is in the interior of the closed domain bounded by $\mathcal{B}^{\mu}$ if and only if $\nu>\mu$.

The supplementary angle of $\nu \in(0, \pi)$ is denoted by $\bar{\nu}=\pi-\nu$, and $\mathbf{u}(\varphi)$ is the unit vector $(\cos \varphi, \sin \varphi)$. Turning the vector $\mathbf{u}(\varphi)$ anticlockwise by $\pi / 2$ gives the unit vector $\mathbf{u}^{\perp}(\varphi):=\mathbf{u}(\varphi+\pi / 2)=(-\sin \varphi, \cos \varphi)$.

Let $p: \mathbb{S}^{1} \rightarrow \mathbb{R}_{+}$be the support function of a convex body $\mathcal{B}$ whose boundary does not contain a segment. Then $p$ is differentiable and the curve

$$
\mathbf{r}(\varphi)=p(\varphi) \mathbf{u}(\varphi)+\dot{p}(\varphi) \mathbf{u}^{\perp}(\varphi)
$$

is obviously closed and has tangent $x \cos \varphi+y \sin \varphi=p(\varphi)$, whence it parametrizes the boundary $\partial \mathcal{B}$ of $\mathcal{B}$.

Lemma 2.1. The $\nu$-isoptic of a convex body $\mathcal{B}$ is continuous at its points. It is differentiable at those of its points that are not collinear with any segment in the boundary $\partial \mathcal{B}$. 
Proof. Let $p$ be the support function of the convex body $\mathcal{B}$. Any point $\mathbf{r}_{\mathcal{B}}^{\nu}$ of the $\nu$-isoptic $\mathcal{B}^{\nu}$ is the intersection of the tangents

$$
\begin{aligned}
p(\varphi) & =x \cos \varphi+y \sin \varphi, \\
p(\varphi+\bar{\nu}) & =x \cos (\varphi+\bar{\nu})+y \sin (\varphi+\bar{\nu}) .
\end{aligned}
$$

Solving the system of these equations leads to

$$
\begin{aligned}
& x=\frac{p(\varphi) \sin (\varphi+\bar{\nu})-p(\varphi+\bar{\nu}) \sin \varphi}{\cos \varphi \sin (\varphi+\bar{\nu})-\sin \varphi \cos (\varphi+\bar{\nu})}=\frac{p(\varphi) \sin (\varphi+\bar{\nu})-p(\varphi+\bar{\nu}) \sin \varphi}{\sin \bar{\nu}}, \\
& y=\frac{p(\varphi) \cos (\varphi+\bar{\nu})-p(\varphi+\bar{\nu}) \cos \varphi}{\sin \varphi \cos (\varphi+\bar{\nu})-\cos \varphi \sin (\varphi+\bar{\nu})}=\frac{p(\varphi+\bar{\nu}) \cos \varphi-p(\varphi) \cos (\varphi+\bar{\nu})}{\sin \bar{\nu}},
\end{aligned}
$$

and hence

$$
\mathbf{r}_{\mathcal{B}}^{\nu}(\varphi)=\frac{1}{\sin \bar{\nu}}\left(p(\varphi+\bar{\nu}) \mathbf{u}^{\perp}(\varphi)-p(\varphi) \mathbf{u}^{\perp}(\varphi+\bar{\nu})\right)
$$

This proves the theorem.

Let $\mathcal{B}$ and $\mathcal{G}$ be convex bodies with support functions $p_{\mathcal{B}}$ and $p_{\mathcal{G}}$, respectively. If their $\nu$-isoptics coincide, that is $\mathcal{B}^{\nu} \equiv \mathcal{G}^{\nu}$, then there is a function $\xi: \mathbb{S}^{1} \rightarrow \mathbb{S}^{1}$ so that $\varphi \mapsto \varphi+\xi(\varphi)$ is bijective on $\mathbb{S}^{1} \rightarrow \mathbb{S}^{1}$, and $\mathbf{r}_{\mathcal{G}}^{\nu}(\varphi)=\mathbf{r}_{\mathcal{B}}^{\nu}(\varphi+\xi(\varphi))$, that, by (2.2), means

$$
\begin{aligned}
p_{\mathcal{G}}(\varphi+\bar{\nu}) & \mathbf{u}(\varphi)-p_{\mathcal{G}}(\varphi) \mathbf{u}(\varphi+\bar{\nu}) \\
& =p_{\mathcal{B}}(\varphi+\xi(\varphi)+\bar{\nu}) \mathbf{u}(\varphi+\xi(\varphi))-p_{\mathcal{B}}(\varphi+\xi(\varphi)) \mathbf{u}(\varphi+\xi(\varphi)+\bar{\nu})
\end{aligned}
$$

This equation translates to the system of equations

$$
\begin{aligned}
& p_{\mathcal{G}}(\varphi+\bar{\nu}) \cos (\varphi)-p_{\mathcal{G}}(\varphi) \cos (\varphi+\bar{\nu}) \\
&=p_{\mathcal{B}}(\varphi+\xi(\varphi)+\bar{\nu}) \cos (\varphi+\xi(\varphi))-p_{\mathcal{B}}(\varphi+\xi(\varphi)) \cos (\varphi+\xi(\varphi)+\bar{\nu}), \\
& p_{\mathcal{G}}(\varphi+\bar{\nu}) \sin (\varphi)-p_{\mathcal{G}}(\varphi) \sin (\varphi+\bar{\nu}) \\
&= p_{\mathcal{B}}(\varphi+\xi(\varphi)+\bar{\nu}) \sin (\varphi+\xi(\varphi))-p_{\mathcal{B}}(\varphi+\xi(\varphi)) \sin (\varphi+\xi(\varphi)+\bar{\nu}) .
\end{aligned}
$$

which has the only solution

$$
\begin{aligned}
p_{\mathcal{G}}(\varphi) \sin (\bar{\nu}) & =-p_{\mathcal{B}}(\varphi+\xi(\varphi)+\bar{\nu}) \sin (\xi(\varphi))+p_{\mathcal{B}}(\varphi+\xi(\varphi)) \sin (\xi(\varphi)+\bar{\nu}), \\
p_{\mathcal{G}}(\varphi+\bar{\nu}) \sin (\bar{\nu}) & =p_{\mathcal{B}}(\varphi+\xi(\varphi)+\bar{\nu}) \sin (\bar{\nu}-\xi(\varphi))+p_{\mathcal{B}}(\varphi+\xi(\varphi)) \sin (\xi(\varphi)) .
\end{aligned}
$$

These equations are consistent if and only if

$$
\begin{aligned}
p_{\mathcal{B}}(\varphi+\xi(\varphi)) \sin (\xi(\varphi))-p_{\mathcal{B}}(\varphi+\xi(\varphi)+\bar{\nu}) \sin (\xi(\varphi)-\bar{\nu}) \\
=p_{\mathcal{B}}(\varphi+\bar{\nu}+\xi(\varphi+\bar{\nu})) \sin (\xi(\varphi+\bar{\nu})+\bar{\nu})- \\
-p_{\mathcal{B}}(\varphi+\bar{\nu}+\xi(\varphi+\bar{\nu})+\bar{\nu}) \sin (\xi(\varphi+\bar{\nu}))
\end{aligned}
$$

With these we have just proved the following result.

Beiträge zur Alg. und Geom., 53 (2012), 281-294.

(c) Á. Kurusa 
Theorem 2.2. A convex body $\mathcal{B}$ has a $\nu$-ghost if and only if the functional consistency equation (2.4) has a solution $\xi$ such that $\varphi \mapsto \varphi+\xi(\varphi)$ is bijective on $\mathbb{S}^{1} \rightarrow \mathbb{S}^{1}$, and

$$
p(\varphi):=\frac{1}{\sin (\bar{\nu})}\left(p_{\mathcal{B}}(\varphi+\xi(\varphi)) \sin (\xi(\varphi)+\bar{\nu})-p_{\mathcal{B}}(\varphi+\xi(\varphi)+\bar{\nu}) \sin (\xi(\varphi))\right)
$$

is the support function of a convex body.

If the support function of an oval is twice differentiable then the parametrization $\mathbf{r}_{\mathcal{B}}^{\nu}: \mathbb{S}^{1} \rightarrow \mathbb{R}^{2}$ of $\mathcal{B}^{\nu}$ as given by (2.2) is also twice differentiable. This allows one to simplify the search for an ovoid ghost of an oval. In fact, exactly this is done in Green's paper [1] for constant $p_{\mathcal{B}}{ }^{(2: 1)}$, but elementary geometry also offers interesting $(\pi / 2)$-ghosts (orthoghosts): ellipses are well known to subtend the angle $\pi / 2$ at the points of their great circle.
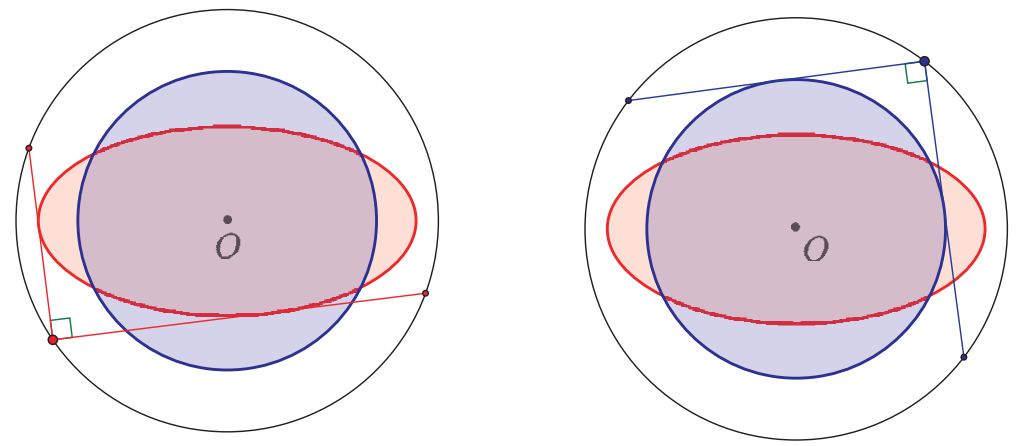

However our next result shows that this is the only case of ghosts among ellipses.

Theorem 2.3. If two ellipses have a common isoptic (it may belong to different angles), then either they coincide or that isoptic is a circle.

Proof. As it can be found in [10] (see [4] for a proof), the $\nu$-isoptic of the ellipse

$$
\mathcal{E}_{a, b}: \quad \frac{x^{2}}{a^{2}}+\frac{y^{2}}{b^{2}}=1
$$

is

$$
\mathcal{E}_{a, b}^{\nu}: \quad\left(x^{2}+y^{2}-a^{2}-b^{2}\right)^{2}=4\left(a^{2} y^{2}+b^{2} x^{2}-b^{2} a^{2}\right) \cot ^{2} \nu
$$

$\overline{(2: 1)}$ A straightforward computation with trigonometric functions shows that $\xi(\varphi)=$ $\varepsilon \sin (n \varphi)$ fulfils the conditions of Theorem 2.2 for any small $\varepsilon>0$.

Beiträge zur Alg. und Geom., 53 (2012), 281-294.

(C) Á. Kurusa 
Since this $\nu$-isoptic is symmetric to the $x$ - and $y$-axis, it may have a further axis of symmetry only through the origin. Suppose that for some $\alpha \in(0, \pi / 2)$ the line $y=x \tan (\alpha / 2)$ is an axis of symmetry of $\mathcal{E}_{a, b}^{\nu}$. Then

$$
\begin{aligned}
\left((x \cos \alpha-y \sin \alpha)^{2}\right. & \left.+(x \sin \alpha+y \cos \alpha)^{2}-a^{2}-b^{2}\right)^{2} \\
& =4\left(a^{2}(x \sin \alpha+y \cos \alpha)^{2}+b^{2}(x \cos \alpha-y \sin \alpha)^{2}-b^{2} a^{2}\right) \cot ^{2} \nu
\end{aligned}
$$

is also an equation of $\mathcal{E}_{a, b}^{\nu}$, which is equivalent to

$$
\begin{aligned}
& \left(x^{2}+y^{2}-a^{2}-b^{2}\right)^{2} \\
& =4\left(a^{2} y^{2}+b^{2} x^{2}+\left(b^{2}-a^{2}\right)\left(y^{2} \sin ^{2} \alpha-x^{2} \sin ^{2} \alpha-2 x y \cos \alpha \sin \alpha\right)-b^{2} a^{2}\right) \cot ^{2} \nu .
\end{aligned}
$$

By comparing this with (2.6), we obtain that

(a) either $\cot \nu=0$, i.e., $\mathcal{E}_{a, b}^{\nu}$ is the circle $x^{2}+y^{2}=a^{2}+b^{2}$ and $\nu=\pi / 2$,

(b) or $a=b$, i.e., $\mathcal{E}_{a, b}$ and $\mathcal{E}_{a, b}^{\nu}$ are the circles with radius $a=b$ and $a \sqrt{2}=b \sqrt{2}$, respectively.

This implies that if two ellipses have a common isoptic, then either that isoptic is a circle or the ellipses have the same axes of symmetries.

If the ellipses have the same two axes of symmetries, then the equations

$$
\begin{array}{ll}
\mathcal{E}_{a, b}^{\nu}: & \left(x^{2}+y^{2}-a^{2}-b^{2}\right)^{2}=4\left(a^{2} y^{2}+b^{2} x^{2}-b^{2} a^{2}\right) \cot ^{2} \nu \\
\mathcal{E}_{c, d}^{\mu}: & \left(x^{2}+y^{2}-c^{2}-d^{2}\right)^{2}=4\left(c^{2} y^{2}+d^{2} x^{2}-c^{2} d^{2}\right) \cot ^{2} \mu
\end{array}
$$

of their common isoptic $\mathcal{E}_{a, b}^{\nu} \equiv \mathcal{E}_{c, d}^{\mu}$ are equivalent. Therefore, if $x=0$, then the equations

$$
\left(y^{2}-b^{2}-a^{2}\right)^{2}=4 a^{2}\left(y^{2}-b^{2}\right) \cot ^{2} \nu \text { and }\left(y^{2}-d^{2}-c^{2}\right)^{2}=4 c^{2}\left(y^{2}-d^{2}\right) \cot ^{2} \mu
$$

must have the same solutions for $y^{2}$, which gives

$$
b^{2}+a^{2}\left(1+2 \cot ^{2} \nu \pm \sqrt{\left(1+2 \cot ^{2} \nu\right)^{2}-1}\right)=y^{2}=d^{2}+c^{2}\left(\frac{1 \pm \cos \mu}{\sin \mu}\right)^{2}
$$

In the same way, if $y=0$, then the common solutions for $x^{2}$ must be the same, and because of the form of the equations, these solutions have the same form as (2.7), but with $a$ exchanged to $b$, and $c$ exchanged to $d$. Therefore we have

$$
\begin{aligned}
& b^{2}+a^{2}\left(\frac{1 \pm \cos \nu}{\sin \nu}\right)^{2}=d^{2}+c^{2}\left(\frac{1 \pm \cos \mu}{\sin \mu}\right)^{2} \\
& a^{2}+b^{2}\left(\frac{1 \pm \cos \nu}{\sin \nu}\right)^{2}=c^{2}+d^{2}\left(\frac{1 \pm \cos \mu}{\sin \mu}\right)^{2}
\end{aligned}
$$

Beiträge zur Alg. und Geom., 53 (2012), 281-294.

(c) Á. Kurusa 
If $x^{2}=y^{2}$, then the equations

$$
\begin{aligned}
& \left(2 x^{2}-b^{2}-a^{2}\right)^{2}=4\left(\left(a^{2}+b^{2}\right) x^{2}-a^{2} b^{2}\right) \cot ^{2} \nu \\
& \left(2 x^{2}-d^{2}-c^{2}\right)^{2}=4\left(\left(c^{2}+d^{2}\right) x^{2}-c^{2} d^{2}\right) \cot ^{2} \mu
\end{aligned}
$$

must have the same solutions for $x^{2}$, that means

$$
\begin{aligned}
& \frac{1 / 2}{\sin ^{2} \nu}\left(a^{2}+b^{2} \pm \sqrt{\left(a^{2}+b^{2}\right)^{2}-\left(\left(a^{2}+b^{2}\right)^{2}+4 a^{2} b^{2} \cot ^{2} \nu\right) \sin ^{4} \nu}\right) \\
& =x^{2}=\frac{1 / 2}{\sin ^{2} \mu}\left(c^{2}+d^{2} \pm|\sin \mu \cos \mu|\left|c^{2}-d^{2}\right|\right)
\end{aligned}
$$

If $a=b$ or $c=d$, then the isoptic is a circle, which proves the statement.

If $a \neq b$ and $c \neq d$, one can eliminate the variables $a, b, c, d$ from equation (2.10) via formulas (2.8) and (2.9), which leads to $\tan (\nu / 2)=\tan (\mu / 2)$, i.e., $\nu=\mu$.

If $\nu=\mu$, equations (2.8) and (2.9) imply that either $c=a$ and $b=d$, i.e., the ellipses coincide, or $(1 \pm \cos \nu)^{2}=\sin ^{2} \nu$, i.e., $\nu=\pi / 2$ and so the isoptic is a circle.

The theorem is proved.

We note that if $\mathcal{E}_{a, b}^{\nu}$ is a circle, i.e., $y^{2}+x^{2}$ is a constant, then the left-hand side of (2.6) is a constant, hence either $\cot \nu=0$, i.e., $\nu=\pi / 2$, or also $a^{2} y^{2}+b^{2} x^{2}$ is a constant. As $\mathcal{E}_{a, b}^{\nu}$ has infinite points, the latter case implies $a^{2}=b^{2}$, i.e., $\mathcal{E}_{a, b}$ is a circle.

\section{General uniqueness}

To generalize the uniqueness part of Green's result we introduce the homeomorphisms $\chi_{\mathcal{B}, \nu}^{ \pm}: \mathcal{B}^{\nu} \rightarrow \mathcal{B}^{\nu}$ and $h_{\mathcal{B}, \nu}^{ \pm}: \mathbb{S}^{1} \rightarrow \mathbb{S}^{1}$.

Let $\ell_{\mathcal{B}}(\mathbf{n})$ be the tangent of the convex body $\mathcal{B}$ that is orthogonal to the unit vector $\mathbf{n}$ and separates the interior of $\mathcal{B}$ from $\mathbf{n}$. Let $T_{\mathcal{B}}(\mathbf{n})$ be a touching point of the tangent $\ell_{\mathcal{B}}(\mathbf{n})$, i.e., $T_{\mathcal{B}}(\mathbf{n}) \in \ell_{\mathcal{B}}(\mathbf{n}) \cap \mathcal{B}^{\nu}$.

Since $\mathcal{B}^{\nu}$ is star-shaped with respect to each point of the convex body $\mathcal{B}, \ell_{\mathcal{B}}(\mathbf{n})$ intersects $\mathcal{B}^{\nu}$ in exactly two points. Let the function $P_{\mathcal{B}, \nu}^{+}: \mathbb{S}^{1} \rightarrow \mathcal{B}^{\nu}$ be such that $P_{\mathcal{B}, \nu}^{+}(\mathbf{n}) \in \ell_{\mathcal{B}}(\mathbf{n})$, and $\overrightarrow{T_{\mathcal{B}}(\mathbf{n}) P_{\mathcal{B}, \nu}^{+}(\mathbf{n})}$ makes a positive base of the plane with $\mathbf{n}$. By $P_{\mathcal{B}, \nu}^{-}$we mean the same but with negative base.

We define the maps $\chi_{\mathcal{B}, \nu}^{ \pm}: \mathcal{B}^{\nu} \rightarrow \mathcal{B}^{\nu}$ so that $\chi_{\mathcal{B}, \nu}^{ \pm}(P)$ is the point $Q \neq P$ of the tangent $\ell_{\mathcal{B}}(\mathbf{n})$ that goes through $P=P_{\mathcal{B}, \nu}^{\mp}(\mathbf{n})$. These maps are homeomorphisms, because each tangent of $\mathcal{B}$ meets exactly two points of $\mathcal{B}^{\nu}$, and $P_{\mathcal{B}, \nu}^{ \pm}$are 
homeomorphisms. We call the homeomorphisms $\chi_{\mathcal{B}, \nu, \pm}$ the right (left, respectively) $\nu$-homeomorphism of $\mathcal{B}$ on $\mathcal{B}^{\nu}$. Obviously $\chi_{\mathcal{B}, \nu}^{+} \circ \chi_{\mathcal{B}, \nu}^{\bar{L}}=\chi_{\mathcal{B}, \nu}^{-} \circ \chi_{\mathcal{B}, \nu}^{+}$is the identity map on $\mathcal{B}^{\nu}$.

We define the maps $h_{\mathcal{B}, \nu}^{ \pm}: \mathbb{S}^{1} \rightarrow \mathbb{S}^{1}$ so that $h_{\mathcal{B}, \nu}^{ \pm}(\mathbf{n})$ is the outer normal vector $\mathbf{n}^{\prime} \neq \mathbf{n}$ of the tangent $\ell_{\mathcal{B}}\left(\mathbf{n}^{\prime}\right)$ that goes through $P_{\mathcal{B}, \nu}^{ \pm}(\mathbf{n})$. These maps are again homeomorphisms, because each point outside $\mathcal{B}$ meets exactly two tangents of $\mathcal{B}$, and $P_{\mathcal{B}, \nu}^{\mp}$ are homeomorphisms. We call the homeomorphisms $h_{\mathcal{B}, \nu}^{ \pm}$the right (left, respectively) $\nu$-homeomorphism of $\mathcal{B}$ on $\mathbb{S}^{1}$. Obviously $h_{\mathcal{B}, \nu}^{+} \circ h_{\mathcal{B}, \nu}^{-}=h_{\mathcal{B}, \nu}^{-} \circ h_{\mathcal{B}, \nu}^{+}$is the identity map on $\mathbb{S}^{1}$.

Theorem 3.1. Let $\bar{\nu} / \pi$ be an irrational or a rational number with even numerator in its lowest terms. If $\mathcal{B}_{1}^{\nu} \equiv \mathcal{B}_{2}^{\nu}$ for the convex bodies $\mathcal{B}_{1}$ and $\mathcal{B}_{2}$, then $\mathcal{B}_{1} \equiv \mathcal{B}_{2}$.

Proof. The convex bodies $\mathcal{B}_{1}$ and $\mathcal{B}_{2}$ have common tangents, because otherwise one of them would contain the other one, and therefore would subtend bigger angle at every points. Furthermore, $\mathcal{B}_{1} \cap \mathcal{B}_{2} \neq \emptyset$ by the following indirect reasoning. If $\mathcal{B}_{1} \cap \mathcal{B}_{2}=\emptyset$, then $\mathcal{B}_{1}$ and $\mathcal{B}_{2}$ have four common tangents. Two of these common tangents, say $\ell_{3}$ and $\ell_{4}$, meets in a point inside the convex hull of $\mathcal{B}_{1} \cup \mathcal{B}_{2}$. The other two common tangents, say $\ell_{1}$ and $\ell_{2}$, meet in a point $P$, that may be at the infinity. Choose the point $Q \in \ell_{1} \cap \mathcal{B}_{1}^{\nu}$ so that the segment $\overline{P Q}$ contains $\left(\mathcal{B}_{1} \cup \mathcal{B}_{2}\right) \cap \ell_{1}$. One of the convex bodies $\mathcal{B}_{1}$ and $\mathcal{B}_{2}$ is on the same side of $\ell_{3}$ as $P$ is, and the other convex body is on the other side of $\ell_{3}$. By renaming, we may assume that $\mathcal{B}_{2}$ is on the same side of $\ell_{3}$ as $P$ is, and $\mathcal{B}_{1}$ is on its other side. This immediately implies that $\mathcal{B}_{1}$ subtends bigger angle at $Q$ than $\mathcal{B}_{2}$ does. This contradicts the fact that $Q \in \mathcal{B}_{1}^{\nu} \equiv \mathcal{B}_{2}^{\nu}$, hence $\mathcal{B}_{1} \cap \mathcal{B}_{2} \neq \emptyset$.

Assume that $\bar{\nu} / \pi$ is irrational and consider a common tangent $\ell_{\mathcal{B}_{1}}\left(\mathbf{n}_{0}\right)$ of $\mathcal{B}_{1}$ and $\mathcal{B}_{2}$. Define the sequence $\mathbf{n}_{i}$ recursively by $\mathbf{n}_{i+1}:=h_{\mathcal{B}_{1}, \nu}^{+}\left(\mathbf{n}_{i}\right)$. Since the tangents $\ell_{\mathcal{B}_{1}}\left(\mathbf{n}_{i+1}\right)$ and $\ell_{\mathcal{B}_{1}}\left(\mathbf{n}_{i}\right)$ intersects each other on $\mathcal{B}_{1}^{\nu} \equiv \mathcal{B}_{2}^{\nu}$, we obtain by induction that each tangent $\ell_{\mathcal{B}_{1}}\left(\mathbf{n}_{i}\right)$ is a common tangent of $\mathcal{B}_{1}$ and $\mathcal{B}_{2}$. As $\bar{\nu} / \pi$ is irrational, the sequence $\mathbf{n}_{i}$ is dense in $\mathbb{S}^{1}$, therefore the support functions $p_{i}: \mathbb{S}^{1} \rightarrow \mathbb{R}_{+}$of the convex bodies $\mathcal{B}_{i}(i=1,2)$ are equal on a dense set. Since the support functions are continuous, we obtain $p_{1}=p_{2}$, hence $\mathcal{B}_{1} \equiv \mathcal{B}_{2}$ as stated.

Now assume that $\bar{\nu} / \pi$ is rational and $\bar{\nu} / \pi=m / n(m, n \in \mathbb{N})$ in its lowest terms.

Choose an arbitrary point $P_{0} \in \mathcal{B}_{j}^{\nu}(j=1,2)$, and define the sequence $P_{i}$ recursively by $P_{i+1}:=\chi_{\mathcal{B}_{j}, \nu}^{+}\left(P_{i}\right)$. Then we have the sequence $\mathbf{n}_{i} \in \mathbb{S}^{1}$ given by $P_{i} P_{i+1}=\ell_{\mathcal{B}}\left(\mathbf{n}_{i}\right)$. Clearly, the angle of $\mathbf{n}_{i}$ to $\mathbf{n}_{i+1}$ is $\bar{\nu}$, therefore

$$
\mathbf{n}_{k n}=\left\{\begin{array}{ll}
\mathbf{n}_{0}, & \text { if } k m \text { is even, and } \\
-\mathbf{n}_{0}, & \text { if } k m \text { is odd, }
\end{array} \quad \text { where } k \in \mathbb{N} .\right.
$$

Beiträge zur Alg. und Geom., 53 (2012), 281-294.

(c) Á. Kurusa 
If $k m$ is even, this shows that $P_{0} P_{1}$ and $P_{k n} P_{k n+1}$ are such parallel tangents of $\mathcal{B}_{j}$ that contain $\mathcal{B}_{j}$ on their same side, hence $P_{0} P_{1}=P_{k n} P_{k n+1}$, i.e., $P_{0}=P_{k n}$.

So, if $m$ is even, then $n$ is odd, therefore $\mathbf{n}_{n}=\mathbf{n}_{0}$ and $P_{0}=P_{n}$, hence $P_{0} P_{1} \cdots P_{n-1}$ is a polygon $\mathcal{P}$ that is inscribed in $\mathcal{B}_{j}^{\nu}$ circumscribed to $\mathcal{B}_{j}$ and has edges of odd number $n \in \mathbb{N}$.

Now we prove that $\mathcal{P}$ is also circumscribed to $\mathcal{B}_{3-j}$.

Now choose a point $B$ in $\mathcal{B}_{1} \cap \mathcal{B}_{2}$, and for any point $P_{k}$ define $\alpha_{k, j}$ and $\beta_{k, j}$ to be the angles $P_{k-1} P_{k} B \angle$ and $B P_{k} P_{k+1} \angle$, respectively. Also we define $\alpha_{k, 3-j}$ and $\beta_{k, 3-j}$ as the angles $\chi_{\overline{\mathcal{B}}_{3-j}, \nu}\left(P_{k}\right) P_{k} B L$ and $B P_{k} \chi_{\mathcal{B}_{3-j}, \nu}^{+}\left(P_{k}\right) L$, respectively. Clearly, we have $\alpha_{k, i}+\beta_{k, i}=\nu$ for all $k \in\{0,1, \ldots, n-1\}$ and $i=1,2$.

If $\beta_{0,1} \neq \beta_{0,2}$, then either $\beta_{0,1}<\beta_{0,2}$ or $\beta_{0,1}>\beta_{0,2}$. Assume $\beta_{0,1}>\beta_{0,2}$. It immediately follows that $\alpha_{1,1}>\alpha_{1,2}$, and therefore $\beta_{1,1}<\beta_{1,2}$ by $\alpha_{1,1}+\beta_{1,1}=\nu=$ $\alpha_{1,2}+\beta_{1,2}$. Iterating this step leads to $0<(-1)^{i}\left(\beta_{i, 1}-\beta_{i, 2}\right)$ for $i \in\{0,1, \ldots, n\}$. But then

$$
0<(-1)^{n}\left(\beta_{n, 1}-\beta_{n, 2}\right)=\beta_{0,2}-\beta_{0,1},
$$

that contradicts the assumption.

If one assumes $\beta_{0,1}<\beta_{0,2}$, then, in the same way, again a contradiction follows, hence we get $\beta_{0,1}=\beta_{0,2}$.

The equation $\beta_{0,1}=\beta_{0,2}$ means that $\mathcal{P}$ is circumscribed also to $\mathcal{B}_{3-j}$.

Since $P_{0}$ was chosen arbitrary on $\mathcal{B}_{j}^{\nu}(j=1,2)$, we have shown that all the tangents of $\mathcal{B}_{j}$ are also tangents of $\mathcal{B}_{3-j}$, which proves the lemma.

As Green's examples show, Theorem 3.1 can not be improved in general.

\section{Distinguishability of convex polygons}

By the inscribed angle theorem, the $\nu$-isoptic $\mathcal{P}^{\nu}$ of a convex polygon $\mathcal{P}$ is the union of circular arcs.

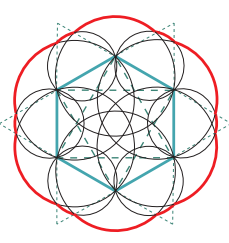

$\nu>\pi / 3$

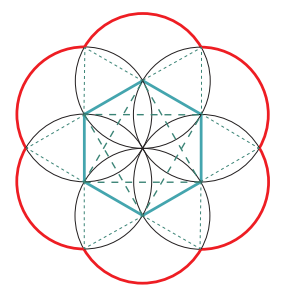

$\nu=\pi / 3$

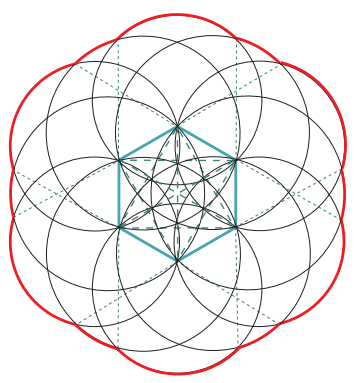

$\nu<\pi / 3$ 
This implies immediately that a convex body $\mathcal{B}$ is not polygonal if its $\nu$-isoptic $\mathcal{B}^{\nu}$ either contains any small piece of a not circular arc or is differentiable at its every points.

Conjecture 4.1. If $\mathcal{B}^{\nu} \equiv \mathcal{P}^{\nu}$ for the convex body $\mathcal{B}$ and a convex polygon $\mathcal{P}$, then $\mathcal{B} \equiv \mathcal{P}$.

This conjecture might be proved via two statements:

(1) If $\mathcal{B}^{\nu} \equiv \mathcal{P}^{\nu}$, then $\mathcal{B}$ is polygonal;

(2) If $\mathcal{P}_{0}^{\nu} \equiv \mathcal{P}_{1}^{\nu}$ for convex polygons $\mathcal{P}_{0}$ and $\mathcal{P}_{1}$, then $\mathcal{P}_{0} \equiv \mathcal{P}_{1}$.

We can prove the second statement.

Theorem 4.2. Two convex polygons with a common connected $\nu$-isoptic coincide.

Proof. Let $\Sigma$ denote the set of the circles of circular arcs in $\mathcal{P}^{\nu}$, and let $\Pi$ be the set of the intersections of these circles. Obviously, the vertices of $\mathcal{P}$ are a subset of $\Pi$.

let $\mathcal{P}^{\nu}$ is the common $\nu$-isoptic of the convex polygons $\mathcal{P}$ and $\mathcal{P}^{\prime}$. As $\mathcal{P}^{\nu}$ is connected, we have $\nu<\min \left(\min _{\mathcal{P}} \alpha, \min _{\mathcal{P}^{\prime}} \alpha\right)$. Neither of these convex polygons can properly contain the others, because then the one that contains the other would subtend bigger angle at all points outside of it.

Thus, there is a common tangent $\ell_{0}$ of $\mathcal{P}$ and $\mathcal{P}^{\prime}$, and therefore there are two further common tangents $\ell_{M}$ and $\ell_{N}$ trough the points $M \neq N$ of $\ell_{0} \cap \mathcal{P}^{\nu}$, respectively.

To prove that $\ell_{0}$ intersects $\mathcal{P}$ and $\mathcal{P}^{\prime}$ in the same edge or vertex, we have to consider several cases.

(a) If $M$ is not an intersection of different circles of $\Sigma$, then all the straight lines of the edges of the convex polygons $\mathcal{P}$ and $\mathcal{P}^{\prime}$ avoid $M$, hence for some vertices $A, B \in \mathcal{P}$ and $A^{\prime}, B^{\prime} \in \mathcal{P}^{\prime}$ we have $\{A\}=\ell_{0} \cap \mathcal{P},\left\{A^{\prime}\right\}=\ell_{0} \cap \mathcal{P}^{\prime},\{B\}=\ell_{M} \cap \mathcal{P}$ and $\left\{B^{\prime}\right\}=\ell_{M} \cap \mathcal{P}^{\prime}$.

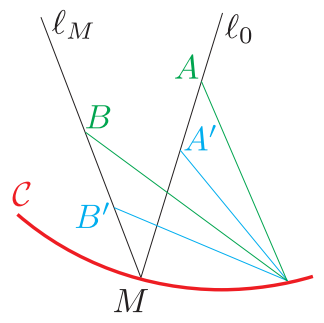

Beiträge zur Alg. und Geom., 53 (2012), 281-294.

(c) Á. Kurusa 
In the other hand, the segments $\overline{A B}$ and $\overline{A^{\prime} B^{\prime}}$ subtend the same constant visual angle $\nu$ on an arc of the circle $\mathcal{C} \in \Sigma$ that contains $M$, hence $A, B, A^{\prime}, B^{\prime} \in \mathcal{C}$ follows and therefore $\ell_{0} \cap \mathcal{P}=\{A\}=\ell_{0} \cap \mathcal{C} \backslash\{M\}=\left\{A^{\prime}\right\}=\ell_{0} \cap \mathcal{P}^{\prime}$, which was to be proved.

(b) If $M$ is an intersection of different circles $\mathcal{C}_{-}$and $\mathcal{C}_{+}$of $\Sigma$, then there are edges of both convex polygons $\mathcal{P}$ and $\mathcal{P}^{\prime}$ that are collinear to $M$, and, since $\ell_{0}$ and $\ell_{M}$ are tangent to both convex polygons, these edges are on $\ell_{0}$ or $\ell_{M}$.

By symmetry in logic, we only need to consider three cases:

(b1) $\overline{A B}=\ell_{0} \cap \mathcal{P}, \overline{A^{\prime} B^{\prime}}=\ell_{0} \cap \mathcal{P}^{\prime},\{C\}=\ell_{M} \cap \mathcal{P}$ and $\left\{C^{\prime}\right\}=\ell_{M} \cap \mathcal{P}^{\prime}$,

(b2) $\overline{A B}=\ell_{0} \cap \mathcal{P},\left\{A^{\prime}\right\}=\ell_{0} \cap \mathcal{P}^{\prime},\{C\}=\ell_{M} \cap \mathcal{P}$ and $\overline{C^{\prime} D^{\prime}}=\ell_{M} \cap \mathcal{P}^{\prime}$,

(b3) $\overline{A B}=\ell_{0} \cap \mathcal{P}, \overline{A^{\prime} B^{\prime}}=\ell_{0} \cap \mathcal{P}^{\prime}, \overline{C D}=\ell_{M} \cap \mathcal{P}$ and $\overline{C^{\prime} D^{\prime}}=\ell_{M} \cap \mathcal{P}^{\prime}$.

In all these cases there is an open neighbourhood $\mathcal{U}_{\mathcal{P}}$ of $M$ that any tangent line of $\mathcal{P}$ through any point of $\mathcal{U}_{\mathcal{P}}$ intersects $\mathcal{P}$ in only one of the points $A$ and $B$. By the same clear reason, there is an open neighbourhood $\mathcal{U}_{\mathcal{P}^{\prime}}$ of $M$ that any tangent line of $\mathcal{P}^{\prime}$ through any point of $\mathcal{U}_{\mathcal{P}^{\prime}}$ intersects $\mathcal{P}^{\prime}$ in only one of the points $C$ and $D$. Let $\mathcal{U}=\mathcal{U}_{\mathcal{P}} \cap \mathcal{U}_{\mathcal{P}^{\prime}}$.

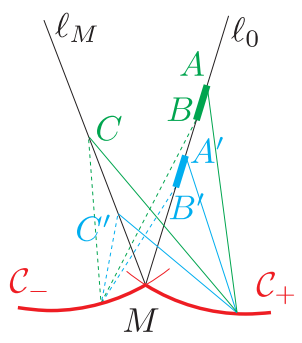

(b1)

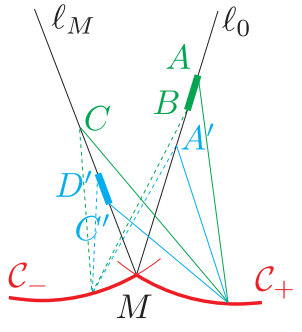

(b2)

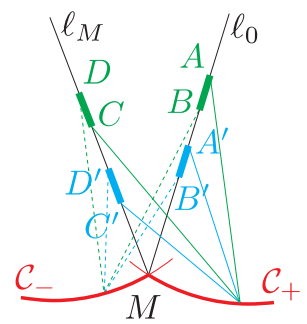

(b3)

Observe, that if a tangent $\ell$ of $\mathcal{P}$ intersects a circular $\operatorname{arc} \mathcal{C} \cap \mathcal{P}^{\nu}$ in two points, then the segment of these intersection points contains the touching points $\ell \cap \mathcal{P}$, because $\mathcal{P}^{\nu}$ is star-shaped from any point of $\mathcal{P}$. Therefore, if a tangent of $\mathcal{P}$ through a point $P \in \mathcal{C}_{+} \cap \mathcal{U}$ cuts $\mathcal{P}$ in $A$, then any tangent that intersects $\mathcal{C}_{-} \cap \mathcal{U}$ cuts $\mathcal{P}$ in $B$, hence by renaming the circles $\mathcal{C}_{-}$and $\mathcal{C}_{+}$of $\Sigma$, we may assume that for any point $P \in \mathcal{C}_{+} \cap \mathcal{U}$ the tangent of $\mathcal{P}$ through $P$ passes $A$, and the tangents of $\mathcal{P}$ through $\mathcal{C}_{-} \cap \mathcal{U}$ contain $B$.

If the point $B^{\prime}$ exists, then by renaming the points $A^{\prime}$ and $B^{\prime}$, we may assume that for any point $P \in \mathcal{C}_{+} \cap \mathcal{U}$ the tangent of $\mathcal{P}$ through $P$ passes $A^{\prime}$, and the tangents of $\mathcal{P}$ through $\mathcal{C}_{-} \cap \mathcal{U}$ contain $B^{\prime}$.

If the point $D$ exists, then by renaming the points $C$ and $D$, we may assume that for any point $P \in \mathcal{C}_{+} \cap \mathcal{U}$ the tangent of $\mathcal{P}$ through $P$ passes $C$, and the tangents of $\mathcal{P}$ through $\mathcal{C}_{-} \cap \mathcal{U}$ contain $D$. 
If the point $D^{\prime}$ exists, then by renaming the points $C^{\prime}$ and $D^{\prime}$, we may assume that for any point $P \in \mathcal{C}_{+} \cap \mathcal{U}$ the tangent of $\mathcal{P}$ through $P$ passes $C^{\prime}$, and the tangents of $\mathcal{P}$ through $\mathcal{C}_{-} \cap \mathcal{U}$ contain $D^{\prime}$.

Thus, the tangents of $\mathcal{P}$ through $\mathcal{C}_{+} \cap \mathcal{U}$ contain $A$, the tangents of $\mathcal{P}^{\prime}$ through $\mathcal{C}_{+} \cap \mathcal{U}$ contain $A^{\prime}$, the tangents of $\mathcal{P}$ through $\mathcal{C}_{-} \cap \mathcal{U}$ contain $C$, and the tangents of $\mathcal{P}^{\prime}$ through $\mathcal{C}_{-} \cap \mathcal{U}$ contain $C^{\prime}$.

Now we are ready to consider the cases (b1), (b2) and (b3) on by one. In all three cases the use of the reasoning of (a) on $\mathcal{C}_{+}$and the points $A, C, A^{\prime}, C^{\prime}$, we conclude $A \equiv A^{\prime}$ and $C \equiv C^{\prime}$. Considering $\mathcal{C}_{-}$in the similar way

(b1) for the points $B, C, B^{\prime}, C^{\prime}$, we infer $B \equiv B^{\prime}$ and $C \equiv C^{\prime}$,

(b2) for the points $B, C, A^{\prime}, D^{\prime}$, we obtain $B \equiv A^{\prime}$ and $C \equiv D^{\prime}$, which is a contradiction, because $A \not \equiv B$, showing that case (b2) can not happen, and

(b3) for the points $B, D, B^{\prime}, D^{\prime}$, we get $B \equiv B^{\prime}$ and $D \equiv D^{\prime}$.

Thus, we conclude the investigations of case (b) by inferring $\ell_{0} \cap \mathcal{P}=\ell_{0} \cap \mathcal{P}^{\prime}$.

As $\ell_{0}$ is an arbitrary common tangent of $\mathcal{P}$ and $\mathcal{P}^{\prime}$, we established that each common tangent intersects $\mathcal{P}$ and $\mathcal{P}^{\prime}$ in the same edge or vertex.

If the vertex $P_{0}$ of $\mathcal{P}$ is outside of $\mathcal{P}^{\prime}$, and no tangent of $\mathcal{P}$ through $P_{0}$ is tangent to $\mathcal{P}^{\prime}$, then straight lines of both edges of $\mathcal{P}$ through $P_{0}$ avoid $\mathcal{P}^{\prime}$. In this case the vertices $P_{-1}$ and $P_{1}$ of $\mathcal{P}$ next to $P_{0}$ are outside of $\mathcal{P}^{\prime}$, and, by the very same reason as for $P_{0}$, either one of them is on a common tangent of $\mathcal{P}$ and $\mathcal{P}^{\prime}$, or the vertices $P_{-2}$ and $P_{2}$ of $\mathcal{P}$ next to $P_{-1}$ and $P_{1}$ are again outside of $\mathcal{P}^{\prime}$. Continuing this reasoning and taking into account that $\mathcal{P}$ has finitely many vertices we conclude that either $\mathcal{P}$ contains $\mathcal{P}^{\prime}$, which is excluded. or there is a vertex $P_{ \pm k}$ of $\mathcal{P}(k \in \mathbb{N})$ outside of $\mathcal{P}^{\prime}$, that is on a common tangent of $\mathcal{P}$ and $\mathcal{P}^{\prime}$. But this latter case contradicts the fact that any common tangent intersects $\mathcal{P}$ and $\mathcal{P}^{\prime}$ in the same edge or vertex.

Therefore no vertex of either $\mathcal{P}$ or $\mathcal{P}^{\prime}$ can be outside of the other one, which proves the theorem.

\section{Distinguishability for rotationally symmetric convex bodies}

As a matter of fact our first result is about reconstructibility of convex bodies rotationally symmetric with respect to the angle $\pi-\nu$.

Proposition 5.1. For any convex body $\mathcal{B}$ and any angle $\nu \in(0, \pi)$ there may be at most one $\nu$-ghost $\mathcal{G}$ of $\mathcal{B}$ that is rotationally symmetric with respect to the angle $\pi-\nu$.

Beiträge zur Alg. und Geom., 53 (2012), 281-294.

(c) Á. Kurusa 
Proof. Place the origin $O$ at the centre of the rotation of $\mathcal{G}$ and define the function $\alpha: \mathbb{S}^{1} \rightarrow \mathbb{S}^{1}$ by the equation $\mathbf{u}(\alpha(\psi))=\mathbf{r}_{\mathcal{B}}^{\nu}(\psi) /\left|\mathbf{r}_{\mathcal{B}}^{\nu}(\psi)\right|$. Then the support function $p_{\mathcal{G}}$ of $\mathcal{G}$ is periodic with period $\bar{\nu}$ and $\alpha$ is bijective.

As the isoptics are star-shaped, for any point $P=\mathbf{r}_{\mathcal{B}}^{\nu}(\psi) \in \mathcal{B}^{\nu}$ there is a unique angle $\varphi$ so that $P=\mathbf{r}_{\mathcal{G}}^{\nu}(\varphi)$, hence, by $(2.2)$, we have

$$
|P|^{2}=\left|\mathbf{r}_{\mathcal{G}}^{\nu}(\varphi)\right|^{2}=p_{\mathcal{G}}^{2}(\varphi) \frac{2(1-\cos \bar{\nu})}{\sin ^{2} \bar{\nu}}=\frac{p_{\mathcal{G}}^{2}(\varphi)}{\cos ^{2}(\bar{\nu} / 2)}
$$

This gives $p_{\mathcal{G}}(\varphi)=|P| \cos (\bar{\nu} / 2)$, which means that $\mathbf{u}(\varphi)$ closes angle $\bar{\nu} / 2$ to the straight line $O P$ which is in direction $\mathbf{u}(\alpha(\psi))$. We conclude, that $p_{\mathcal{G}}(\alpha(\psi)-\bar{\nu} / 2)=$ $\left|\mathbf{r}_{\mathcal{B}}^{\nu}(\psi)\right| \cos (\bar{\nu} / 2)$ for all $\psi$, which, by the bijectivity of $\alpha$, determines $p_{\mathcal{G}}$ on $\mathbb{S}^{1}$. This proves the statement.

Now we are relaxing the condition of rotational symmetry.

Theorem 5.2. The convex body $\mathcal{B}$ has rotational symmetry with respect to the angle $2(\pi-\nu)$ if and only if the length $\left|\mathbf{r}_{\mathcal{B}}^{\nu}\right|$ of the parametrization $\mathbf{r}_{\mathcal{B}}^{\nu}$ of $\mathcal{B}^{\nu}$ is periodic with period $\pi-\nu$.

Proof. Let $\mathcal{B}$ be a convex body of rotational symmetry with respect to the angle $2 \bar{\nu}$, and choose the origin to be the centre of this rotation. Then its support function $p_{\mathcal{B}}$ is periodic with period $2 \bar{\nu}$ and, by $(2.2)$, we have

$$
\begin{aligned}
\left|\mathbf{r}_{\mathcal{B}}^{\nu}(\varphi)\right|^{2} \sin ^{2} \bar{\nu} & =p_{\mathcal{B}}^{2}(\varphi)+p_{\mathcal{B}}^{2}(\varphi+\bar{\nu})-2 p_{\mathcal{B}}(\varphi) p_{\mathcal{B}}(\varphi+\bar{\nu}) \cos \bar{\nu} \\
& =p_{\mathcal{B}}^{2}(\varphi+2 \bar{\nu})+p_{\mathcal{B}}^{2}(\varphi+\bar{\nu})-2 p_{\mathcal{B}}(\varphi+2 \bar{\nu}) p(\varphi+\bar{\nu}) \cos \bar{\nu} \\
& =\left|\mathbf{r}_{\mathcal{B}}^{\nu}(\varphi+\bar{\nu})\right|^{2} \sin ^{2} \bar{\nu}
\end{aligned}
$$

hence $\left|\mathbf{r}_{\mathcal{B}}^{\nu}(\varphi)\right|$ is periodic with period $\bar{\nu}$.

If $\left|\mathbf{r}_{\mathcal{B}}^{\nu}(\varphi)\right|$ is periodic with period $\bar{\nu}$, then $\left|\mathbf{r}_{\mathcal{B}}^{\nu}(\varphi)\right|^{2}=\left|\mathbf{r}_{\mathcal{B}}^{\nu}(\varphi+\bar{\nu})\right|^{2}$, which, by (2.2), implies

$$
0=\left(p_{\mathcal{B}}(\varphi+2 \bar{\nu})-p_{\mathcal{B}}(\varphi)\right)\left(p_{\mathcal{B}}(\varphi+2 \bar{\nu})+p_{\mathcal{B}}(\varphi)-2 p_{\mathcal{B}}(\varphi+\bar{\nu}) \cos \bar{\nu}\right)
$$

By convexity we have

$$
\begin{aligned}
p_{\mathcal{B}}(\varphi & +2 \bar{\nu})+p_{\mathcal{B}}(\varphi) \\
& =\left\langle\mathbf{u}(\varphi+2 \bar{\nu}), \mathbf{r}_{\mathcal{B}}(\varphi+2 \bar{\nu})\right\rangle+\left\langle\mathbf{u}(\varphi), \mathbf{r}_{\mathcal{B}}(\varphi)\right\rangle \\
& \geq\left\langle\mathbf{u}(\varphi+2 \bar{\nu}), \mathbf{r}_{\mathcal{B}}(\varphi+\bar{\nu})\right\rangle+\left\langle\mathbf{u}(\varphi), \mathbf{r}_{\mathcal{B}}(\varphi+\bar{\nu})\right\rangle \\
& =\left\langle\mathbf{u}(\varphi+2 \bar{\nu})+\mathbf{u}(\varphi), p_{\mathcal{B}}(\varphi+\bar{\nu}) \mathbf{u}(\varphi+\bar{\nu})+\dot{p}_{\mathcal{B}}(\varphi+\bar{\nu}) \mathbf{u}^{\perp}(\varphi+\bar{\nu})\right\rangle \\
& =2 p_{\mathcal{B}}(\varphi+\bar{\nu}) \cos \bar{\nu}
\end{aligned}
$$

Beiträge zur Alg. und Geom., 53 (2012), 281-294.

(c) Á. Kurusa 
which is an equality if and only if

$$
\left\langle\mathbf{u}(\varphi+2 \bar{\nu}), \mathbf{r}_{\mathcal{B}}(\varphi+2 \bar{\nu})-\mathbf{r}_{\mathcal{B}}(\varphi+\bar{\nu})\right\rangle=0 \text { and }\left\langle\mathbf{u}(\varphi), \mathbf{r}_{\mathcal{B}}(\varphi+\bar{\nu})-\mathbf{r}_{\mathcal{B}}(\varphi)\right\rangle=0 .
$$

Condition (5.3) means that the point $\mathbf{r}_{\mathcal{B}}(\varphi+\bar{\nu})$ is the intersection of the tangents with normal vectors $\mathbf{u}(\varphi+2 \bar{\nu})$ and $\mathbf{u}(\varphi)$. The number of such points is at most $\pi /(2 \bar{\nu})$, therefore inequality $(5.2)$ is strict except for finitely many $\varphi$. Taking into account that the support function $p_{\mathcal{B}}$ is continuous, this implies $p_{\mathcal{B}}(\varphi+2 \bar{\nu}) \equiv p_{\mathcal{B}}(\varphi)$ by $(5.1)$.

If an isoptic $\mathcal{B}^{\nu}$ is a circle, then $\mathbf{r}_{\mathcal{B}}^{\nu}$ is periodic with period $\pi-\nu$, hence the convex body $\mathcal{B}$ has rotational symmetry with respect to the angle $2(\pi-\nu)^{(5: 2)}$ by our Theorem 5.2.

Lemma 5.3. Let the convex body $\mathcal{B}$ be rotationally symmetric with respect to the angle $2(\pi-\nu)$. If the polygon $\mathcal{P}$ is circumscribed to $\mathcal{B}$ and inscribed in $\mathcal{B}^{\nu}$, then the vertices of $\mathcal{P}$ are on a circle centred at the rotational centre of $\mathcal{B}$ and the lengths of the edges of $\mathcal{P}$ are alternating with two values.

Proof. Let $\mathbf{r}_{\mathcal{B}}^{\nu}(\varphi)$ be a vertex of $\mathcal{P}$. Then $\mathbf{r}_{\mathcal{B}}^{\nu}(\varphi+k \bar{\nu})$ are the vertices of $\mathcal{P}$, where $k \in \mathbb{Z}$. Theorem 5.2 gives then that the vertices are on the circle $\mathcal{C}$ of radius $\left|\mathbf{r}_{\mathcal{B}}^{\nu}(\varphi)\right|$ centred to the origin.

The edges of $\mathcal{P}$ are the chords of $\mathcal{C}$, so the lengths of them are determined by their distances from the origin, which are $p_{\mathcal{B}}(\varphi+k \bar{\nu})$. This alternates according to the parity of $k$, because the period of $p_{\mathcal{B}}$ is $2 \bar{\nu}$.

Theorem 5.4. If the convex bodies $\mathcal{B}$ and $\mathcal{G}$ have only finitely many common tangents, both are rotationally symmetric with respect to the angle $2(\pi-\nu)$, and if they have common $\nu$-isoptic $\mathcal{B}^{\nu} \equiv \mathcal{G}^{\nu}$, then $\mathcal{B}^{\nu}$ is a circle.

Proof. Since neither $\mathcal{G}$ nor $\mathcal{B}$ can contain the other, there are common tangents of $\mathcal{G}$ and $\mathcal{B}$. If a sequence of common tangents has a limit $\ell$ (that is an accumulation point of the set of common tangents), then $\ell$ is a common tangent of $\mathcal{G}$ and $\mathcal{B}$. Therefore the set $\mathcal{L}$ of common tangents is closed.

Let $\ell$ be a common tangent of $\mathcal{G}$ and $\mathcal{B}$ that is isolated in $\mathcal{L}$, and intersects $\mathcal{B}^{\nu}$ in the points $\{P, Q\}=\ell \cap \mathcal{B}^{\nu}$. Assume, that $\mathcal{B}$ is in the halfplane $\mathcal{S}_{\ell}^{-}$of $\ell$, which is to the left of $\overrightarrow{P Q}$.

$\overline{(5: 2)}$ A direct proof of this comes from $\bar{\nu}=\arccos \left(p_{\mathcal{B}}(\varphi) / \varrho\right)+\arccos \left(p_{\mathcal{B}}(\varphi+\bar{\nu}) / \varrho\right)$, where $\varrho>0$ is the radius of the circle $\mathcal{B}^{\nu}$.

Beiträge zur Alg. und Geom., 53 (2012), 281-294.

(c) Á. Kurusa 
Choose a point $P_{0} \in \mathcal{B}^{\nu}$ in the halfplane $\mathcal{S}_{\ell}^{+}$of $\ell$, which is to the right of $\overrightarrow{P Q}$, so that $P_{0}$ is so near to $P$ that no straight line in $\mathcal{L}$ intersects a small neighbourhood of the $\operatorname{arc} \mathcal{B}_{0}^{\nu}$ of $\mathcal{B}^{\nu}$ that connects $P$ to $P_{0}$ (except of course $\ell$ ).

Let $P_{1}$ be the only such point of $\mathcal{B}^{\nu}$ that $P_{0} P_{1}$ is a tangent line of $\mathcal{B}$ that separates $\mathcal{G} \cup \mathcal{B}$ from $Q$. If such point does not exist, then there must exist a unique point $P_{1} \in \mathcal{B}^{\nu}$ so that $P_{0} P_{1}$ is a tangent line of $\mathcal{G}$ that separates $\mathcal{G} \cup \mathcal{B}$ from $Q$.

For simplicity we assume the first case, that is, $P_{1}$ is the only such point of $\mathcal{B}^{\nu}$ that $P_{0} P_{1}$ is a tangent line of $\mathcal{B}, P_{0} P_{1}$ is outside of $\mathcal{G}$ and $\mathcal{B}$ is on the left-hand side of $\overrightarrow{P_{0} P_{1}}$.

Let $P_{2}$ be the only such point of $\mathcal{B}^{\nu}$ that $P_{1} P_{2}$ is a tangent line of $\mathcal{G}$ and $\mathcal{G}$ is on the right-hand side of $\overrightarrow{P_{1} P_{2}}$. Then, obviously, $P_{1} P_{2}$ intersects $\mathcal{B}$.

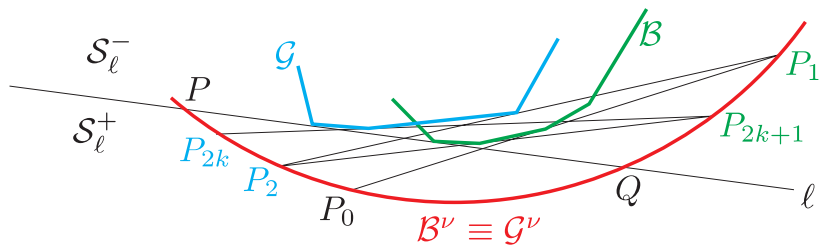

Given the point $P_{2 k}$ on $\mathcal{B}^{\nu}$ for some $k \in \mathbb{N}$, let $P_{2 k+1}$ be the only such point of $\mathcal{B}^{\nu}$ that $P_{2 k} P_{2 k+1}$ is a tangent line of $\mathcal{B}$ and $\mathcal{B}$ is on the left-hand side of $\overrightarrow{P_{2 k} P_{2 k+1}}$. Then, obviously, $P_{2 k} P_{2 k+1}$ is outside of $\mathcal{G}$. Next, let $P_{2 k+2}$ be the only such point of $\mathcal{B}^{\nu}$ that $P_{2 k+1} P_{2 k+2}$ is a tangent line of $\mathcal{G}$ and $\mathcal{G}$ is on the right-hand side of $\overrightarrow{P_{2 k+1} P_{2 k+2}}$. Then, again obviously, $P_{2 k+1} P_{2 k+2}$ intersects $\mathcal{B}$.

This gives an infinite sequence $\left\{P_{i}: i \in \mathbb{N}\right\}$ of points on $\mathcal{B}^{\nu}$ so that the points of even indexes are in $\mathcal{S}_{\ell}^{+}$and the points of odd indexes are in $\mathcal{S}_{\ell}^{-}$.

Since both $\mathcal{B}$ and $\mathcal{G}$ are rotationally symmetric with respect to the angle $2 \bar{\nu}$, Lemma 5.3 implies $\left|P_{2 i}\right|=\left|P_{2 i+1}\right|$ and $\left|P_{2 i+1}\right|=\left|P_{2 i+2}\right|$, respectively. Thus, we obtain $\left|P_{2 i}\right|=\left|P_{2 i+1}\right|=|P|$ for all $i \in \mathbb{N}$.

Let $0<\gamma_{i}<\pi / 2$ be the angle of the straight line $\ell_{i}=P_{i} P_{i+1}$ to $\ell$. It is an immediate consequence of the construction of the points $\left\{P_{i}: i \in \mathbb{N}\right\}$, that the sequence $\gamma_{i}$ is strictly decreasing, hence there exists $0 \leq \gamma_{\infty}=\lim _{i \rightarrow \infty} \gamma_{i}<$ $\pi / 2$. Since every straight line $\ell_{i}$ intersects the segment $\overline{P Q}$, we infer the existence of a common tangent $\ell_{\infty}=\lim _{i \rightarrow \infty} \ell_{i}$. But $\ell_{0}$ was chosen so that $\mathcal{B}_{0}^{\nu}$ is not intersected by any common tangent, so $\ell_{\infty}=\ell$ follows, hence $\lim _{i \rightarrow \infty} P_{2 i}=P$ and $\lim _{i \rightarrow \infty} P_{2 i+1}=Q$.

We have proved that $\mathcal{B}^{\nu}$ is a circular arc near any endpoint of any isolated common tangent and the radii of the circles of these arcs are equal.

Since $\mathcal{L}$ is finite, every common tangents of $\mathcal{B}$ and $\mathcal{G}$ are isolated.

Beiträge zur Alg. und Geom., 53 (2012), 281-294.

(c) Á. Kurusa 
Take a maximal arc $\mathcal{B}_{\max }^{\nu}$ of $\mathcal{B}^{\nu}$ that is not intersected by any common tangent of $\mathcal{B}$ and $\mathcal{G}$. Let $P$ and $P^{\prime}$ be the two closing points of $\mathcal{B}_{\text {max }}^{\nu}$. Then there are unique common tangents $\ell, \ell^{\prime} \in \mathcal{L}$ through $P$ and $P^{\prime}$, respectively, so that $\ell$ and $\ell^{\prime}$ separate $P^{\prime}$ and $P$, respectively, from $\mathcal{B} \cup \mathcal{G}$. We define the points $Q$ and $Q^{\prime}$ by $\ell \cap \mathcal{B}^{\nu}=\{P, Q\}$ and $\ell^{\prime} \cap \mathcal{B}^{\nu}=\left\{P^{\prime}, Q^{\prime}\right\}$.

Let $P_{0}$ be any point of $\mathcal{B}_{\max }^{\nu}$, and let $P_{i}$ be the sequence of points generated from $P_{0}$ as above so that $P_{2 i} P_{2 i+1}$ separates $\mathcal{B} \cup \mathcal{G}$ from $\left\{Q, P^{\prime}\right\}$. Then the angles $0<\gamma_{i}<\pi / 2$ of the straight lines $P_{2 i} P_{2 i+1}$ to $P Q$ decreases monotonously again by the construction, so the sequence of the straight lines $P_{2 i} P_{2 i+1}$ converges, and therefore it converges to $P Q$. Thus any point of $\mathcal{B}_{\max }^{\nu}$ is on the circle of the circular arc of $\mathcal{B}^{\nu}$ near $P$, hence $\mathcal{B}_{\max }^{\nu}$ is a circular arc.

This proves the theorem completely.

An obvious, but interesting statement follows.

Theorem 5.5. Two convex bodies with rotational symmetry with respect to the angle $2(\pi-\nu)$ having the same noncircular $\nu$-isoptic coincide.

According to W. Wunderlich's result mentioned already in the introduction, the condition of rotational symmetry is crucial in this theorem. However Theorem 2.3 shows that the result may be valid with some other conditions as well.

\section{More isoptics}

Referring to Green's result, Klamkin in 1988 raised the question "If two isoptic curves of a convex domain are concentric circles, is it then also a concentric circle?" (see [2]), which was affirmatively answered by J.C.C. Nitsche [8] two years later.

It is most natural to broaden Klamkin's question as follows:

How many isoptics of a convex body are necessary to permit its exact reconstruction?

To the best knowledge of the author, not any answer to this question - except, of course, the ones of Green and Nitsche - was published by now. The following specialized version of [3, Theorem 5] gives the best answer the author could give by now. 
Theorem 6.1. If $\left\{\nu_{i}\right\}_{i \in \mathbb{N}}$ is a strictly decreasing sequence of angles in $(0, \pi)$, and the $\nu_{i}$-isoptics of two convex bodies coincide for all $i$, then the convex bodies also coincide.

Nitsche's result and the additional conditions imposable by Theorem 3.1 offer hope for improvement of Theorem 6.1, but the author sees nothing that would support a better result in general.

\section{References}

[1] J. W. Green, Sets subtending a constant angle on a circle, Duke Math. J., 17 (1950), 263-267.

[2] M. S. Klamkin, Conjectured isoptic characterization of a circle, Amer. Math. Monthly, 95 (1988), 845.

[3] Á. Kurusa, The shadow picture problem for nonintersecting curves, Geom. Dedicata, 59 (1996), 113-125.

[4] Á. Kurusa, Kúpszeletek izoptikusai, Polygon, 19:1 (2011), 27-46 (in Hungarian).

[5] H. Martini and W. Mozgawa, An integral formula related to inner isoptics, Rendic. Sem. Mat. Univ. Padova, 125 (2011), 39-49.

[6] A. Miernowski and W. Mozgawa, On some geometric condition for convexity of isoptics, Rend. Sem. Mat. Univ. Pol. Torino, 55 (1997), 93-98.

[7] W. Mozgawa, Integral formulas related to ovals, Beiträge Algebra Geom., 50 (2009), 555-561.

[8] J. C. C. Nitsche, Isoptic characterization of a circle, (Proof of a conjecture of M.S. Klamkin), Amer. Math. Monthly, 97 (1990), 45-47.

[9] W. Wunderlich, Kurven mit isoptischen Ellipse, Monatshefte Math., 75 (1971), 346-362.

[10] R. C. Yates, A Handbook on Curves and their Properties, J. W. Edwards, Ann. Arbor, 1947, pp. 138-140.

Á. KurusA, Bolyai Institute, University of Szeged, Aradi vértanúk tere 1., H-6720 Szeged, Hungary; e-mail: kurusa@math.u-szeged.hu 